\title{
Attitudes towards vaccines, intent to vaccinate and the relationship with COVID-19 vaccination rates in individuals with schizophrenia
}

Running Title: Attitudes to COVID-19 vaccination in schizophrenia

Stéphane Raffard ${ }^{1,2}$, Sophie Bayard ${ }^{1}$, Margot Eisenblaetter ${ }^{1}$, Jérôme Attal $^{2}$, Christelle Andrieu $^{3,}$ Isabelle Chereau ${ }^{4,5}$, Guillaume Fond ${ }^{3}$, Sylvain leignier ${ }^{5}$, Jasmina Mallet ${ }^{6,7,8}$, Philippe $\operatorname{Tattard}^{2}$, Mathieu Urbach ${ }^{9,10}$, David Misdrahi ${ }^{11}$, Yasmine Laraki ${ }^{1,2}$, Delphine Capdevielle ${ }^{2,12}$

${ }^{1}$ Univ Paul Valéry Montpellier 3, EPSYLON EA 4556, Montpellier, France; ${ }^{2}$ University Department of Adult Psychiatry, CHU Montpellier, Montpellier, France; ${ }^{3}$ Pole psychiatrie, centre expert depression résistante et schizophrénie FondaMental, CHU La Conception, Marseille, France; Aix-Marseille university, CEReSS-health service research and quality of life center, 27, boulevard Jean-Moulin, 13005 Marseille, France; ${ }^{4}$ CHU Clermont-Ferrand, Service de psychiatrie B, Université Clermont Auvergne, Clermont-Ferrand, France; ${ }^{5}$ Centre Référent de Réhabilitation Psychosociale et de Remédiation Cognitive (C3R), CH Alpes Isère, France; ${ }^{6}$ AP-HP ; Department of Psychiatry, Louis Mourier Hospital, Colombes, France $;{ }^{7}$ Inserm UMR1266, Institute of Psychiatry and Neuroscience of Paris, University Paris Descartes, France; ${ }^{8}$ Université Paris Diderot, Sorbonne Paris Cité, Faculté de médecine, France; ${ }^{9}$ Versailles Hospital, Department of Adult Psychiatry and Addictology, Centre Hospitalier de Versailles, 177 rue de Versailles, 78157 Le Chesnay, France,; ${ }^{10}$ Dis APDevPsy-CESP, INSERM UMR1018, University of Paris-Saclay, University of Versailles Saint-Quentin-En-Yvelines 94807, Villejuif, France; ${ }^{11}$ Department of Adult Psychiatry, Charles Perrens Hospital, Bordeaux, France, University of Bordeaux, Laboratory of Nutrition and Integrative Neurobiology (UMR INRA 1286), France; ${ }^{12}$ IGF, Univ. Montpellier, CNRS, 
medRxiv preprint doi: https://doi.org/10.1101/2022.01.12.22269167; this version posted January 17, 2022. The copyright holder for this preprint (which was not certified by peer review) is the author/funder, who has granted medRxiv a license to display the preprint in perpetuity. It is made available under a CC-BY-NC-ND 4.0 International license .

INSERM, Montpellier, France

*To whom correspondence should be addressed; Laboratory Epsylon, EA 4556, Montpellier University 3, University Department of Adult Psychiatry, 39 Avenue Charles

Flahault 34295 Montpellier Cedex 5, France. Tel.:+33 4673397 02; fax: +33 467339660 /

Email: s-raffard@chu-montpellier.fr

\section{Abstract}


Background: People with schizophrenia are at high risk for developing severe COVID-19 outcomes but recent evidence suggests that this population have lower vaccination rates than the general population. This gap in vaccination rates could be explained by attitudinal and structural barriers.

Aims: This study explored the role of potential attitudinal barriers by comparing schizophrenia patients with participants from the general population regarding COVID-19 vaccination rates, general attitudes towards vaccines, and willingness to take a COVID-19 vaccine.

Method: We conducted a cross-sectional study between April 2021 and October 2021. 100 schizophrenia patients and 72 controls were recruited. Attitudes towards vaccines were assessed with the Vaccination Attitudes Examination Scale. COVID-19 vaccination rates, sociodemographic, clinical and health-related variables were also assessed.

Results: In our study, the proportion of individuals with schizophrenia were under-vaccinated despite similar general attitudes towards vaccination and higher willingness to be vaccinated against COVID-19 compared to the participants from the general population. In patients, negative attitudes toward vaccines was related to higher level of negative psychotic symptoms and higher level of paranoid ideation. As a whole, participants with more negative attitudes towards vaccines were less likely to be vaccinated against COVID-19 and had lower levels of trust in institutions.

Conclusions: Vaccine hesitancy does not appear to be a major barrier for COVID-19 vaccine uptake amongst people with schizophrenia. This study suggests that disparities in COVID-19 vaccination rates in schizophrenia do not seem related to attitudinal but rather structural barriers. 
Keywords: COVID-19, attitudes, vaccination, schizophrenia

\section{Significant outcomes}

- In both groups, non-vaccinated individuals had higher general negative attitudes towards vaccines.

- $\quad$ People with schizophrenia were under-vaccinated (64\% versus $77.8 \%)$ despite similar general attitudes towards vaccination and higher willingness to be vaccinated in non vaccinated against COVID-19 compared to the controls (41.7\% versus $21.5 \%)$.

- General positive attitudes towards vaccines were negatively associated with persecutory ideation and negative symptoms in patients.

\section{Limitations}

- $\quad$ Our two sample groups sere composed exclusively of French individuals, potentially limiting the generalizability of our results.

- The cross-sectional design of our study.

\section{Introduction}

The worldwide pandemic caused by the COVID-19 (SARS-CoV-2) has strongly impacted individuals' lives with dramatic economical and global health consequences. More than 250 million cases and 5 million deaths worldwide were reported as of November 30 , 2021 (WHO). An accumulating body of research pointed out that individuals with severe 
mental illnesses, including schizophrenia spectrum disorders, mood, and substance use disorders are at higher risk of developing serious COVID-19 outcomes, including hospitalizations and death(1,2,3,4). Among the different public health measures that have been implemented to curb this health crisis, the recent development of effective vaccines constitutes a decisive step in the successful control of this pandemic. As such, promoting vaccination access in people with schizophrenia is a public health priority (5). However, and despite the demonstrated effectiveness of vaccines for reducing the mortality and morbidity of COVID-19 in this population, a cross-sectional and a longitudinal study reported an association between this pathology and lower vaccination rates $(6,7)$. These studies are in line with what has already been observed in other preventive vaccines like the influenza vaccine $(8,9)$.

Based on past global experiences from infectious disease control, researchers have identified two types of barriers for vaccination: structural and attitudinal (10). Structural barriers correspond to «systemic issues that may limit the ability of individual persons to access a vaccine service ».(10) Regarding schizophrenia several structural barriers have been identified including cost, limited access to online health information, awareness of services, or absence of medical recommendation $(5,9)$.

The second type of barriers for vaccination refers to attitudes which can be defined as a set of emotions, beliefs, and behaviors toward a particular object, person, thing, or event $(11,12)$. Similarly to all existing vaccines, it has been shown that the most important attitudinal barriers to receiving a COVID-19 vaccine are a general mistrust in the benefits and safety of vaccines as well as concerns about their unforeseen secondary effects (13). As a result, vaccination attitudes are considered as multidimensional rather than a unidimensional construct $(10,14)$. Recently and within the context of the COVID-19 pandemic, two largescale studies have shown that both conspiracy beliefs and persecutory ideas, two 
psychological features that are highly present in individuals with schizophrenia, were positively associated with COVID-19 vaccine hesitancy and resistance in the general population $(15,16)$. Finally, our research team recently showed that impaired competence to consent to a COVID-19 vaccine was associated with lower vaccination rates in people with schizophrenia (17).

However, the large amount of literature on attitudes towards vaccination focuses on the general population and very few studies have explored these attitudes in the context of severe mental disorders. The first and only study that has assessed attitudes towards vaccination (excluding the COVID-19 pandemic) showed that individuals with schizophrenia perceived vaccination against influenza as effective as non-clinical controls, but more risky for adverse reactions (18). Regarding COVID-19 vaccination two recent studies in China and Israel showed that individuals with various mental disorders had similar positive attitudes for the COVID-19 vaccine compared to the general population $(19,20)$. However, the majority of their sample comprised of people diagnosed with anxiety disorders and depression and only a small sample $(\mathrm{N}=16)$ of individuals with psychotic disorders $(19,20)$. In contrast to these two studies, a third study showed that individuals with schizophrenia had more negative attitudes than the general population towards COVID-19 vaccination (21). However, in their study attitudes towards vaccination was measured using a single question "Do you intend to be vaccinated against COVID-19 in the future?" which rather than assessing attitudes this question measures the intention to be vaccinated (21). As a result, attitudes of people with schizophrenia spectrum disorders regarding vaccination remains largely unexplored. Moreover, understanding how attitudes are related to both intent to be vaccinated and vaccination rates has not yet been investigated in people with schizophrenia despite evidence that COVID-19 vaccination is crucial for this population $(5,22)$. 
Consequently, the main aims of this study were: 1) to assess general attitudes towards vaccines using a multidimensional scale in individuals with schizophrenia compared to a nonclinical control group, 2) to determine vaccination rates as a function of general attitudes towards vaccines in individuals with schizophrenia, and 3) to investigate the clinical, cognitive and psychological determinants of attitudes toward vaccines in general and COVID19 vaccination in individuals with schizophrenia.

\section{Methods}

Sample

One-hundred participants with a diagnosis of schizophrenia were recruited using the same protocol from four independent sites from France between April 2021 to October 2021: CHU Montpellier ( $\mathrm{N}=81)$, CHU Clermont-Ferrand ( $\mathrm{N}=8$ ), AP-HP Marseille $(\mathrm{N}=7), \mathrm{CH}$ Alpes Isère $(\mathrm{N}=3)$, Versailles Hospital $(\mathrm{N}=1)$. Inclusion criteria for patients were: (a) age between 18 and 65 years, (b) a DSM-5 diagnosis of schizophrenia, (c) adequate proficiency in French. Exclusion criteria for all participants were: (a) known neurological disease, (b) history of learning disability/developmental disorder, or (c) substance abuse in the past month (other than cannabis or tobacco). Seventy-two controls were recruited from the general population with an additional exclusion criterion: no personal lifetime history diagnosis of a psychotic or affective disorder. To ensure the absence of psychotic disorders in the nonclinical participants, we used the $7^{\text {th }}$ version of the DSM-5 Mini-International Neuropsychiatric Interview. Non-clinical participants who had a family member with either bipolar or schizophrenia disorder were also excluded. The demographic data are shown in Table 1.

Insert Table 1.

\section{Assessments}


Clinical and cognitive assessments. The Positive and Negative Syndrome Scale (PANSS) is a 30-item scale designed to assess specific symptomatology in psychotic disorders in individuals diagnosed with a schizophrenia disorder (23). Each item is based on a 1 to 7 rating-scale and overall scores include a positive, negative, general psychopathology and total score. Paranoid ideations were assessed in both groups using the short version of the Green Paranoid Thoughts Scale (GPTS) which has recently been validated in French (24). This version of the GTPS comprises of 8 items based on a 5-point rating scale ranging from 1 (not at all) to 5 for (totally). Global cognitive functioning was assessed in both groups using the Montreal Cognitive Assessment (MoCA, 25).

Vaccination status and health-related indicators. COVID-19 vaccination status was recorded for all participants as either no vaccination or first dose of vaccine/fully vaccinated.

Participants were also asked if they or any of their immediate family members had any of the following underlying health conditions: diabetes, lung disease, or cardiovascular conditions. We also recorded whether the participants had a confirmed or suspected case of COVID-19 infection.

Attitudes toward vaccines. Negative general attitudes towards vaccines were measured using the 12-item Vaccination Attitudes Examination Scale (VAX, 14). All participants were asked to focus on vaccines in general rather than specifically on a COVID-19 vaccine. Responses on the $\mathrm{VAX}$ are rated on a six-point scale ranging from 1 (strongly agree) to 6 (strongly disagree). Four subscale-scores were calculated: (1) mistrust of vaccine benefit, (2) worries about unforeseen future effects, (3) concerns about commercial profiteering, and (4) preference for natural immunity. Note that higher scores indicate higher negative attitudes 
towards vaccines. The French version of the VAX also showed good internal consistency, construct validity and test-retest reliability (26).

Intent to take a COVID-19 vaccine. For the unvaccinated participants we also measured the intent to take a COVID-19 vaccine. We used a single item question taken from the IPSOSWEF COVID-19 VACCINE GLOBAL SURVEY JANUARY 2021 - GLOBAL PR: “If a vaccine for COVID-19 is available for me, I would get it". Participants were asked to respond to this item on a 4 point-scale ranging from 1 (strongly disagree) to 4 (strongly agree).

Trust in institutions. In order to evaluate the trust in institutions, we used the same methodology than Murphy et al. (16). Participants were asked to indicate the level of trust they had in political parties, the parliament, the government, the police, the legal system, scientists, doctors and other health professionals. This questionnaire is composed of 7 items and responses were scored on a five-point Likert scale ranging from "do not trust at all" (1) to

" completely trust" (5). A total score was computed whereby higher scores indicated more trust in institutions.

Procedure

Following a thorough explanation of the study, all participants provided informed and written consent before participating. Sociodemographic and treatment information was collected and participants were then administered the measures, for a duration of approximately one hour. This study was conducted in accordance to the ethical standards described by the Medical Research Involving Human Subjects Act (WMO) and was approved by the hospital's institutional review board (IRB ID: 202100768). 
Statistical analysis

Statistical analyses were performed with the Jamovi statistical computer software [The jamovi project (2021). jamovi. (Version 1.6) Retrieved from http://www.jamovi.org]. Means and standard deviations were computed for continuous variables and categorical variables were expressed in percentages. Absolute values for skewness and kurtosis greater than 3 and 20 , respectively, were used to test normality (27). The winsorizing method was used to process any outlier scores on questionnaires. This method consists of replacing outlier scores by assigning a value on a unit smaller or larger than the next most extreme (non-outlier) score in the distribution (28).

Group differences in demographic variables, clinical variables, and scores on the questionnaires were analyzed using independent-sample Student's t-test and analysis of variance for parametric variables. Chi-square tests were used for categorical variables. In addition, we calculated the eta squared $\eta 2$, the Cohen's d' and the Phi-coefficient as measures of effect size. The effect size was considered small $(\eta 2=0.01 ; d=0.2 ;$ Phi $=0.10)$, medium $(\eta 2=0.06 ; d=0.5 ;$ Phi $=0.30)$, or large $(\eta 2=0.14 ; d=0.8 ;$ Phi $=0.5)$. To test for the potential relationships between variables in the whole sample and per group, we used Pearson correlations with rs of $0.10,0.30$, and 0.50 defined as small, medium, and large effect size, respectively. All analyses were conducted with a significance threshold of $\alpha \leq 0.05$, twotailed.

\section{Results}

Preliminary analyses

All data from questionnaires had satisfactory skewness and kurtosis values, suggesting a normal distribution. In the total sample and for all items of our variables of interest $(n=$ $1548)$, only 10 values were identified as univariate outliers $(10 / 1548) * 100=0.64 \%$. 


\section{Demographic variables}

As documented in Table 1, patients were older than controls $(\mathrm{P}<.001, \mathrm{~d}=-0.63)$ and overall had a lower level of education $(\mathrm{P}<.001, \mathrm{~d}=0.56)$. No significant group difference was noted in terms of gender $(\mathrm{P}=.15)$.

\section{Clinical and cognitive assessments}

All patients were under antipsychotic treatment. Based on the GPTS results, patients reported higher level of paranoid ideations compared to the controls $(P=.02, \mathrm{~d}=-0.33)$. The clinical group also showed lower global cognitive functioning on the MOCA compared to the non-clinical group $(\mathrm{P}<.001, \mathrm{~d}=0.93)$ (Table 1).

Vaccination status, health-related indicators, and intention to vaccinate

A statistical trend was noted with respect to the proportion of vaccinated participants, showing lower rates of vaccination in patients compared to controls (respectively, 64\% versus $77.8 \%, \mathrm{P}=.07$, Phi $=0.15)$. Note that neither age, education, nor global cognitive functioning was associated with vaccination status in either groups (all Ps $>.20$ ). The proportion of participants infected or suspected of having been infected with COVID-19 was not significantly different in the two groups $(\mathrm{P}=.15)$. Patients reported significantly higher levels of underlying health conditions compared to controls $(\mathrm{P}=.01)$. In both groups, no association was found between vaccination status and health-related indicators (all Ps > .40). Finally, among the non-vaccinated participants, the proportion of individuals intending to be vaccinated was significantly higher in patients compared to controls $(\mathrm{P}=.03$, Phi $=0.29)$.

Vaccination status, demographic variables, health-related indicators, and clinical and cognitive assessments

In both patients and in controls, no association was found between vaccination status, demographic variables (i.e., age, education and gender) and health-related indicators (all Ps < 
.13). In patients, vaccination status was not significantly associated with global cognitive functioning $(\mathrm{P}=.34)$, psychotic symptoms $($ all $\mathrm{Ps}>.56)$ nor paranoid ideations $(\mathrm{P}=0.13)$.

Vaccination status, attitudes towards vaccines, trust in institutions, and COVID-19 related questionnaires

Figure 1 illustrates that there was a significant difference in all VAX dimensions based on vaccination status (all Ps $<.01$ ). Non-vaccinated individuals had higher negative attitudes towards vaccines across all VAX dimensions. Furthermore, there was a significant group effect $\left(\mathrm{P}=.004, \eta^{2}=.04\right)$ for the VAX mistrust of vaccine benefit dimension (Figure 1A). Patients reported higher confidence in the benefits of vaccination compared to controls. No group effect was observed for the other three dimensions of the VAX questionnaire (Figures 1B, C and D; all Ps > .22). Interactions were not significant either (Figure 1; all Ps > .10).

Finally, significant association was noted between vaccination status and group in the Trust in institutions questionnaire. Non-vaccinated individuals had lower levels of trust in institutions than did the vaccinated participants $\left(F=5.7, P=.003, \eta^{2}=.05\right.$, respectively, 21.1 $\mathbf{\pm 6 . 7}$ versus $22.7 \quad \mathbf{\pm}$.4). Patients had a significantly higher level of confidence in the institutions than did the controls $\left(\mathrm{F}=9.2, \mathrm{P}=.02, \eta^{2}=0.03\right.$, respectively, $23.1 \pm 4.5$ versus $20.9 \pm 6.5)$. The interaction between vaccination status and group was not significant $(\mathrm{F}=1.7$, $\mathrm{P}=.18)$.

Associations between attitudes towards vaccines, COVID-19 related questionnaires, demographic variables, health-related indicators, and clinical and cognitive assessments

In both patients and controls, no association was found between attitudes toward vaccines, demographic variables and health-related indicators (all Ps > .10). In patients, the 
preference for natural immunity VAX dimension was related to higher level of negative psychotic symptoms $(r=0.31, P=0.01)$ and to higher level of paranoid ideation $(r=0.22, P=$ .02). Higher level of paranoid ideation was also associated with the concerns about commercial profiteering VAX domain $(\mathrm{r}=0.21, \mathrm{P}=.04)$.

In both patients and controls, both the VAX mistrust of vaccine benefit (respectively, $r$ $=-0.39, \mathrm{P}<.001$ versus $\mathrm{r}=-0.32, \mathrm{P}=.005)$ and concerns about commercial profiteering VAX dimensions were related to a lower level of trust in institutions $(r=-0.23, P=.02$ versus $r=-0.51, P<.001)$. In controls only was higher level of worries about unforeseen future effects VAX dimension associated to lower level of trust in institutions $(r=-0.32, \mathrm{P}=.005)$.

\section{Discussion}

Currently there is evidence that individuals with mental disorders, and particularly individuals with schizophrenia, should be prioritized for COVID-19 vaccination as hospitalization and mortality rates are higher in this population in comparison to the general population $(2,7)$. Attitudinal barriers towards vaccination might reduce vaccine uptake in this population but these remain largely unexplored, specifically within the context of the COVID-19 pandemic (22,29).

First, general attitudes towards vaccination as measured by the total score of the VAX scale were similar between patients and controls. Patients and controls did not differ regarding beliefs about unforeseen future effects, concerns about commercial profiteering, and preference for natural immunity. However, patients scored lower on the mistrust of vaccine benefit dimension of the VAX compared to the controls. This indicates that our sample of patients (even though were under-vaccinated) have more positive attitudes regarding vaccination and have a stronger belief that we can rely on vaccines to stop and control the spread of serious infectious diseases than non-clinical participants did. On average, patients 
felt safer after being vaccinated, considered vaccines to be more effective in stopping serious infectious diseases and felt more protected after getting vaccinated compared to controls. Our results are partially in accordance with Maguire et al. who found that in their sample of individuals with schizophrenia, patients considered the vaccine for influenza as being as effective than the control group, but the patients were more likely to perceive vaccination as being risky for an adverse reaction (18). One explanation could be that, contrarily to the influenza vaccines, the government and the media throughout the world have heavily promoted COVID-19 vaccination. Due to the severity of this pandemic, the development of vaccines and vaccination campaigns progressed rapidly worldwide potentially contributing to an overall increase in vaccine confidence. In addition, our results indicate that individuals with schizophrenia (and individuals with other mental disorders) do not necessarily have more negative attitudes towards vaccines than the general population $13,30,31$ ). This differs from what has been shown in other COVID-19 at-risk populations (e.g., ethnic minority groups and individuals with lower incomes or education) $(30,32,33)$.

Second, a trend was observed in terms of the proportion of vaccinated participants which was lower in patients in comparison to the non-clinical controls (respectively, 64\% versus $77.8 \%, P=.07)$. Though COVID-19 vaccination rates in people diagnosed with severe mental disorders are to date largely unknown in most countries, a recent study in Israël, which included a large sample of patients, indicated that people with schizophrenia are under-vaccinated for COVID-19 compared to the general population (7). Very importantly, among non-vaccinated participants, a significantly higher proportion of patients had the intention to be vaccinated compared to non-clinical controls. In other words, despite similar general positive attitudes towards vaccines and higher willingness to be vaccinated (for the non-vaccinated individuals), rates of vaccination for COVID-19 was lower in our patient group. 
Two types of barriers to vaccination have been described in the literature: structural (e.g. poor internet access, shorter life expectancy, geographical distance from vaccination sites, living in more deprived areas) and attitudinal (i.e. beliefs or perceptions that impact the willingness to be vaccinated) $(7,10,22,34)$. Overall our results suggest that structural barriers rather than individual barriers might explain the lower vaccination rates in individuals with schizophrenia. This is in line with the fact that although people with schizophrenia are at higher risk than other populations in developing some physical diseases, they are rarely considered as a priority group to receive a vaccination. However, research has shown that when this population is correctly informed, the willingness to be vaccinated is high (see similar results regarding pneumonia and Influenza) $(8,35)$

Third, we found that in both groups, non-vaccinated individuals had higher general negative attitudes towards vaccines across all VAX dimensions. Even though a causal inference between general attitudes towards vaccination and vaccination rates cannot be inferred, this is the first time that such an association is found in schizophrenia. Additionally, this suggests that attitudes toward COVID-19 vaccination might influence vaccination coverage rates, which is the case for other types of vaccines such as influenza vaccines (36). Further studies using a longitudinal design are needed to draw definitive conclusions.

Fourth, correlational analyses found negative relationships between positive attitudes toward vaccination and negative symptoms and paranoid ideations, measured using the PANSS and the GPTS, respectively. The symptomatology of individuals with schizophrenia should thus be considered when assessing confidence and intent towards vaccination. The association between paranoid ideation and general negative attitudes towards vaccination in schizophrenia is in accordance with previous studies conducted in the general population whereby similar associations between conspiracy beliefs, persecutory beliefs and higher resistance to a COVID-19 vaccine was found $(15,16)$. The association between negative 
symptoms and vaccination hesitancy appears however difficult to explain and remains elusive. Nevertheless, the desires for restrictions to ease and life to return to normal (e.g. go to the cinema, travel) has been found as the most common reasons for positive attitudes towards COVID-19 vaccine $(37,38)$. It is likely that the desire to perform such activities do not constitute a strong enough factor in reducing vaccine hesitancy in people with schizophrenia with high levels of negative symptoms. For example, studies have recurrently shown that negative symptoms, and specifically apathy-anhedonia, lead to a reduced capacity in experiencing and anticipating pleasure from activities (39). A reduction in interpersonal interactions and goal-directed behaviors have also been noted in this population. This could also explain the association found between negative symptoms and vaccination hesitancy.

The current study had several limitations that restrict the interpretation and generalizability of the results. The main limitation is the cross-sectional design of our study. Even though we found an association between negative attitudes and lower vaccination rates in both groups, we cannot conclude a causal relationship between these two variables. Future studies with a longitudinal design are needed. Second, our sample group was composed exclusively of French individuals, limiting the generalizability of our results. There is evidence that in Europe, and particularly in France, trust towards vaccination remains low compared to other continents (38). Replication of the present study in other countries would allow for a better understanding of attitudes towards vaccination in different populations (including in individuals with mental illnesses).

Conclusions. The present study found that individuals with schizophrenia have similar general attitudes towards vaccines than the general population. The non-vaccinated patients also endorsed a higher willingness to be vaccinated against COVID-19 compared to the controls. However as a whole, people with schizophrenia were under-vaccinated in our 
sample suggesting the presence of structural barriers rather than attitudinal barriers in getting vaccinated within this clinical population. Developing targeted vaccination programs is urgently needed for this population $(40,41)$.

Acknowledgements. None

\section{Statement of Ethics}

Participants in the study were treated in accordance with international ethical standards, including APA standards of ethics, and was approved by the hospital's institutional review board (IRB ID: 202100768).

\section{Conflict of Interest Statement}

No authors declare any possible conflicts of interest.

\section{Funding sources}

This work was founded by a 2021 ANR Grant, (SCHIZOVAC; ANR-21-COVR-0017)

\section{Data availability}

The anonymised dataset is available from the corresponding author on reasonable request

\section{References}


1. Tzur Bitan D, Krieger I, Kridin K, Komantscher D, Scheinman Y, Weinstein O, Cohen

AD, Cicurel AA, Feingold D. COVID-19 Prevalence and Mortality Among

Schizophrenia Patients: A Large-Scale Retrospective Cohort Study. Schizophr Bull. 2021;47:1211-1217. doi: 10.1093/schbul/sbab012

2. Fond G, Pauly V, Leone M, Llorca PM, Orleans V, Loundou A, Lancon C, Auquier P, Baumstarck K, Boyer L. Disparities in Intensive Care Unit Admission and

Mortality Among Patients With Schizophrenia and COVID-19: A National

Cohort Study. Schizophr Bull. 2021;47:624-634. doi: 10.1093/schbul/sbaa158.

3. Jeon HL, Kwon JS, Park SH, Shin JY. Association of mental disorders with SARS-CoV-2 infection and severe health outcomes: nationwide cohort study. Br J Psychiatry. 2021 Jan 7:1-8. doi: 10.1192/bjp.2020.251.

4. Wang Q, Xu R, Volkow ND. Increased risk of COVID-19 infection and mortality in people with mental disorders: analysis from electronic health records in the United States.

World Psychiatry. 2021;20:124-130. doi: 10.1002/wps.20806

5. Warren N, Kisely S, Siskind D. Maximising the uptake of a COVID-19 vaccine in people with severe mental illness: a public health priority. JAMA Psychiatry 2020;

78:589-590. doi: 10.1001/jamapsychiatry.2020.4396

6. Tzur Bitan D. Patients with schizophrenia are under-vaccinated for COVID-19: a report from Israel. World Psychiatry. 2021;20:300-301. doi: 10.1002/wps.20874.

7. Tzur Bitan D, Kridin K, Cohen AD, Weinstein O. COVID-19 hospitalisation, mortality, vaccination, and postvaccination trends among people with schizophrenia in

Israel: a longitudinal cohort study. Lancet Psychiatry. 2021;8:901-908. doi:

$10.1016 /$ S2215-0366(21)00256-X. 
8. Lorenz RA, Norris MM, Norton LC, Westrick SC. Factors associated with influenza vaccination decisions among patients with mental illness. Int J Psychiatry Med

2013; 46: $1-13$.

9. Miles LW, Williams N, Luthy KE, Eden L. Adult Vaccination Rates in the Mentally Ill Population: An Outpatient Improvement Project J Am Psychiatr Nurses Assoc. ;26:172-180. doi: 10.1177/1078390319831763

10. Zhang Y, Fisk RJ. Barriers to vaccination for coronavirus disease 2019 (COVID-19) control: experience from the United States. Glob Health J. 2021 Mar;5:51-55.

doi: 10.1016/j.glohj.2021.02.005

11. Breckler, S. J. (1984). Empirical validation of affect, behavior, and cognition as distinct

components of attitude. J. Pers. Soc. Psychol. 47, 1191-1205. https://doi.org/ 10.1037/0022-3514.47.6.1191

12. Chaiklin H. Attitudes, behavior, and social practice. J. Soc. \& Soc. Welfare. 2011;38:31

13. Paul E, Steptoe A, Fancourt D. Attitudes towards vaccines and intention to vaccinate against COVID-19: Implications for public health communications.

Lancet Reg Health Eur. 2021;1:100012. doi: 10.1016/j.lanepe.2020.100012.

14. Martin LR Petrie KJ. Understanding the Dimensions of Anti-Vaccination Attitudes: the Vaccination Attitudes Examination (VAX) Scale. Ann Behav Med. 2017; 51: $652-660$.

15. Freeman D, Waite F, Rosebrock L, Petit A, Causier C, East A, Jenner L, Teale AL, Carr L, Mulhall S, Bold E, Lambe S. Coronavirus conspiracy beliefs, mistrust, and compliance with government guidelines in England. Psychol Med. 2020;21:1-13. doi: 10.1017S0033291720001890 Study. 
16. Murphy J, Vallières F, Bentall RP, Shevlin M, McBride O, Hartman TK, McKay R,

Bennett K, Mason L, Gibson-Miller J, Levita L, Martinez AP, Stocks TVA,

Karatzias

T, Hyland P. Psychological characteristics associated with COVID-19

vaccine hesitancy and resistance in Ireland and the United Kingdom.

Nat Commun. 2021;

12(1):29. doi: 10.1038 s41467-020-20226-9.

17. Raffard S, Bayard S, Eisenblaetter M, Tattard P, Attal J, Laraki Y, \& Capdevielle D.

Mental capacity to consent to COVID 19 vaccination in individuals

with schizophrenia : an explorative study (submitted for publication).

18. Maguire PA, Reay RE, Looi JC. Nothing to sneeze at - uptake of protective measures against an influenza pandemic by people with schizophrenia: willingness and perceived barriers. Australas Psychiatry.

2019;27:171-178.doi:10.1177/1039856218815748

19. Hao F, Wang B, Tan W, Husain SF, McIntyre RS, Tang X, Zhang L, Han X, Jiang L, Chew NWS, Tan BY, Tran B, Zhang Z, Vu GL, Vu GT, Ho R, Ho CS, Sharma VK. Attitudes toward COVID-19 vaccination and willingness to pay: comparison of people with and without mental disorders in China. BJPsych Open. 2021;7(5):e146. doi: 10.1192/bjo.2021.979

20 Danenberg R, Shemesh S, Tzur Bitan D, Maoz H, Saker T, Dror C, Hertzberg L, Bloch Y. Attitudes of patients with severe mental illness towards COVID-19 vaccinations: A preliminary report from a public psychiatric hospital. J Psychiatr Res. 2021

;143:16-20. doi: 10.1016/j.jpsychires.2021.08.020

21. Bai W, Cai H, Jin Y, Zhang Q, Cheung T, Su Z, Tang YL, Ng CH, Xiang YT. COVID-19 vaccine hesitancy in community-dwelling and hospitalized patients with severe mental illness. Psychol Med. 2021 Dec 17:1-6. doi: 10.1017/S0033291721004918. 
22. Mazereel V, Van Assche K, Detraux J, De Hert M. COVID-19 vaccination for people with severe mental illness: why, what, and how? Lancet

Psychiatry $2021 ; 8(10): 860-861$.

23. Kay, S.R., Fiszbein, A., Opler, L.A., 1987. The positive and negative syndrome scale (PANSS) for schizophrenia. Schizophr. Bull. 13 (2), 261-276.

24. Bianchi R, Verkuilen J. "Green et al. Paranoid Thoughts Scale": French validation and development of a brief version. Pers. Individ. Differ. 2021; 171, 110554.

25. Nasreddine ZS, Phillips NA, Bédirian V, Charbonneau S, Whitehead V, Collin I, Cummings JL, Chertkow H. The Montreal Cognitive Assessment, MoCA: a brief screening tool for mild cognitive impairment. J Am Geriatr Soc. 2005 ; 53:695-9. doi: 10.1111/j.1532-5415.2005.53221.x

26. Eisenblaetter M, Madiouni, C., Laraki Y, Capdevielle D, \& Raffard S. French validation of the Vaccination Attitudes Examination (VAX) Scale (submitted for publication).

27. Weston R, PaulA G. "A brief guide to structural equation modeling." The counseling psychologist 2006; 34:719-751.

28. Tabachnik BG, Fidell SL. "Multicollinearity and singularity." Using multivariate statistics. Boston: Pearson Education Inc 2013; 2: 88-91.

29. Suhas S. COVID 19 vaccination of persons with schizophrenia in India - Need for imperative action! Schizophr Res. 2021;231:49-50. doi: 10.1016/ j.schres.2021.03.003

30. Stead M, Jessop C, Angus K, Bedford H, Ussher M, Ford A, Eadie D, MacGregor A, Hunt K, MacKintosh AM. National survey of attitudes towards and intentions to 
vaccinate against COVID-19: implications for communications. BMJ Open.

2021;11(10):e055085. doi: 10.1136/bmjopen-2021-055085.

31. Jefsen OH, Kølbæk P, Gil Y, Speed M, Dinesen PT, Sønderskov KM, Østergaard

SD.COVID-19 vaccine willingness amongst patients with mental illness

compared with the general population. Acta Neuropsychiatr. 2021;33(5):273-276.

doi: $\quad 10.1017 /$ neu.2021.15

32. AlShurman BA, Khan AF, Mac C, Majeed M, Butt ZA. What Demographic, Social, and Contextual Factors Influence the Intention to Use COVID-19 Vaccines: A

Scoping Review. Int J Environ Res Public Health. 2021;18(17):9342. doi:

10.3390/ ijerph18179342.

33. Robinson E, Jones A, Lesser I, Daly M. International estimates of intended uptake and refusal of COVID-19 vaccines: A rapid systematic review and meta-analysis

of large nationally representative samples. Vaccine. 2021;39:2024-2034. doi: 10.1016j.vaccine.2021.02.005.

34. Njoku A, Joseph M, Felix R. Changing the Narrative: Structural Barriers and Racial and Ethnic Inequities in COVID-19 Vaccination. Int J Environ Res Public Health. ;18(18):9904. doi: 10.3390/ijerph18189904.

35. Seminog OO, Goldacre MJ. Risk of pneumonia and pneumococcal disease in people with severe mental illness: English record linkage studies. Thorax. 2013;68(2):171-

6. doi: 10.1136/thoraxjnl-2012-202480.

36. Wang Q, Yue N, Zheng M, Wang D, Duan C, Yu X, Zhang X, Bao C, Jin H. Influenza vaccination coverage of population and the factors influencing influenza vaccination in mainland China: A meta-analysis. Vaccine.

2018;36:7262-7269. doi: 10.1016/j.vaccine.2018.10.045. 
37. Cordina M, Lauri MA, Lauri J. Attitudes towards COVID-19 vaccination, vaccine hesitancy and intention to take the vaccine. Pharm Pract (Granada). 2021 JanMar;19(1):2317. doi: 10.18549 PharmPract.2021.1.2317.

38. de Figueiredo A, Simas C, Karafillakis E, Paterson P, Larson HJ. Mapping global trends in vaccine confidence and investigating barriers to vaccine uptake: a large-scale retrospective temporal modelling study. Lancet. 2020;396(10255):898-908. doi: 10.1016/S0140-6736(20)31558-0.

39. Fervaha G, Foussias G, Agid O, Remington G. Impact of primary negative symptoms on functional outcomes in schizophrenia. Eur Psychiatry. 2014 ;29:449-55. doi: 10.1016/j.eurpsy.2014.01.007. Epub 2014 Mar 14. PMID: 24630742.

40. De Hert M, Mazereel V, Detraux J, Van Assche K. Prioritising COVID-19 vaccination for people with severe mental illness. World Psychiatry 2021; 20: 54-55.

41. Mazereel V, Vanbrabant T, Desplenter F, De Hert M. COVID-19 vaccine uptake in patients with psychiatric disorders admitted to or residing in a university psychiatric hospital. Lancet Psychiatryx2021;8:860-861.

Table 1. Sociodemographic and clinical characteristics of the sample 


\begin{tabular}{|c|c|c|c|c|}
\hline$N(\%) /$ mean $(\mathrm{SD})$ & $\begin{array}{l}\text { Patients } \\
N=100\end{array}$ & $\begin{array}{l}\text { Controls } \\
N=72\end{array}$ & Statistics & $P$-value \\
\hline \multicolumn{5}{|l|}{ Demographic variables } \\
\hline Age & $40.6(12.4)$ & $32.7(12.6)$ & $t=-4.09$ & $<.001$ \\
\hline Gender, female & $37.5 \%$ & $26 \%$ & $\chi^{2}=2.09$ & .15 \\
\hline Years of scholarship, mean & $12.7(2.7)$ & $14.1(2.2)$ & $t=3.76$ & $<.001$ \\
\hline \multicolumn{5}{|l|}{ Clinical and cognitive assessments } \\
\hline Patients under treatment & $100 \%$ & & & \\
\hline Duration of the disease & $16.5(11.3)$ & & & \\
\hline PANSS total & $63.9(17.7)$ & & & \\
\hline PANSS positive & $13.7(5.7)$ & & & \\
\hline PANSS negative & $16.8(5.6)$ & & & \\
\hline PANSS general psychopathology & $33.2(8.5)$ & & & \\
\hline Green Paranoid Thoughts Scale & $16.1(8.6)$ & $13.4(6.9)$ & $\mathrm{t}=-2.16$ & .02 \\
\hline Montreal Cognitive Assessment & $23.9(4.1)$ & $27.1(2.4)$ & $t=6.33$ & $<.001$ \\
\hline \multicolumn{5}{|c|}{ Vaccination status and health-related indicators } \\
\hline COVID-19 vaccination, yes & $64 \%$ & $77.8 \%$ & $\chi^{2}=3.14$ & .07 \\
\hline COVID-19 infection, yes & $15 \%$ & $23.6 \%$ & $\chi^{2}=2.1$ & .15 \\
\hline Underlying health condition, yes & $22 \%$ & $8.3 \%$ & $\chi^{2}=5.74$ & .01 \\
\hline Intend to take a COVID-19 vaccine ${ }^{1}$ & $41.7 \% \%$ & $12.5 \%$ & $\chi^{2}=4.28$ & .03 \\
\hline
\end{tabular}

Note. PANSS, Positive and Negative Syndrome Scale

${ }^{1}$ Among non-vaccinated participants (patients, $N=36$; controls, $N=16$ ) 